\title{
INTRODUCCIÓN A LA TECNOLOGIA DE MEMBRANAS PARA LA PURIFICACION DE BIOGAS Y ALGUNOS DESARROLLOS RECIENTES
}

\author{
Cristian Cardona Gonzalez¹, Diego Andrés Molina Cardona², Roger Junior González Chevejoni ${ }^{3}$, Oscar \\ Felipe Arbeláez Perez ${ }^{4}$.
}

1 ingeniero Mecánico, Semillero de Investigación en Energía y Medio Ambiente, Universidad Cooperativa de Colombia, cristian.cardonag@campusucc.edu.co.

2 Ingeniero Mecánico, Semillero de Investigación en Energía y Medio Ambiente, Universidad Cooperativa de Colombia, diego.molinac@campusucc.edu.co,

${ }^{3}$ Ingeniero Mecánico, Semillero de Investigación en Energía y Medio Ambiente, Universidad Cooperativa de Colombia, roger.gonzalezc@campusucc.edu.co,

4 Doctor en Ingeniería, Grupo de Investigación Termomec, Universidad Cooperativa de Colombia. oscar.arbelaez@campusucc.edu.co

\section{RESUMEN}

La remoción de $\mathrm{CO}_{2}$ en mezclas de $\mathrm{CO}_{2} / \mathrm{CH}_{4}$ para aumentar el contenido de energía en gas natural o biogás y prevenir problemas de corrosión, ha impulsado el desarrollo del proceso de separación de $\mathrm{CO}_{2}$ utilizando membranas. Las características más relevantes que ofrece la tecnología basada en membranas incluyen la alta eficiencia energética, el costo reducido y el rendimiento altamente flexible. Esta revisión proporciona una descripción de los trabajos reportados desde 2010 hasta 2020 sobre los diferentes tipos de membranas disponibles: poliméricas, inorgánicas y de matriz mixta para el proceso de separación de $\mathrm{CO}_{2} / \mathrm{CH}_{4}$; se reportan las condiciones experimentales y los determinantes primarios del rendimiento y la eficiencia de la separación (permeabilidad de $\mathrm{CO}_{2}$ y selectividad $\mathrm{CO}_{2} / \mathrm{CH}_{4}$ ). Este trabajo ofrece una nueva perspectiva de cada membrana para facilitar una mejor apreciación de su papel en la mejora del rendimiento general del proceso.

Palabras clave: biogás; membranas poliméricas; membranas inorgánicas; membranas de matriz mixta; separación de dióxido de carbono.

Recibido: 27 de enero de 2021. Aceptado: 15 de abril de 2021

Received: January 27, 2021. Accepted: April 15, 2021

DOI: http://dx.doi.org/10.33571/rpolitec.v17n33a6

\section{INTRODUCTION TO MEMBRANE TECHNOLOGY FOR BIOGAS PURIFICATION AND SOME RECENT DEVELOPMENTS}

\begin{abstract}
The remotion of $\mathrm{CO}_{2}$ from $\mathrm{CO}_{2} / \mathrm{CH}_{4}$ mixes to increasing energy content in natural gas or biogas and to prevent corrosion problems, has driven the development of $\mathrm{CO}_{2}$ separation process through membranes. The attractive features offered by this technology include high energy efficiency, reduced cost and highly flexible performance. This review provides an overview of the reported paper from 2010 to 2020 different types of membranes available: polymeric, inorganic and mixed matrix for $\mathrm{CO}_{2} / \mathrm{CH}_{4}$ separation process, experimental conditions and primary determinants of separation performance and efficiency (permeability of $\mathrm{CO}_{2}$ and $\mathrm{CO}_{2} / \mathrm{CH}_{4}$ selectivity). This work would open up a new perspective of each membrane to facilitate a better appreciation of their role in the improvement of overall process performance.
\end{abstract}

Keywords: biogas; polymeric membranes, inorganic membranes; mixed-matrix membrane; $\mathrm{CO}_{2}$ separation

Cómo citar este artículo: C. Cardona-Gonzalez, D.A. Molina-Cardona, R.J. González-Chevejoni, O.F. Arbeláez-Perez. "Introducción a la tecnología de membranas para la purificación de biogas y algunos desarrollos recientes", Revista Politécnica, vol. 17, no.33 pp.76-89, 2021. DOI: http://dx.doi.org/ 10.33571/rpolitec.v17n33a6 


\section{INTRODUCCIÓN}

El consumo de combustibles fósiles está ligado al avance de la industria y de la humanidad, sin embargo éste contribuye al crecimiento en la emisión de gases de efecto invernadero (vapor de agua, clorofluorocarbonos, hidrofluorocarbonos, dióxido de carbono, metano, óxido nitroso), especialmente dióxido de carbono (Figura 1), lo cual genera el aumento en la temperatura global, la reducción de las reservas petroleras y la inestabilidad de los precios de los combustibles [1]. Acorde con la tendencia de las emisiones de dióxido de carbono mostrada en la Figura 1, se prevé que las emisiones de dióxido de carbono aumenten a 43.000 millones de toneladas métricas para 2040, por lo tanto, se necesitan recursos energéticos complementarios que sean accesibles, renovables y de fácil obtención [2].

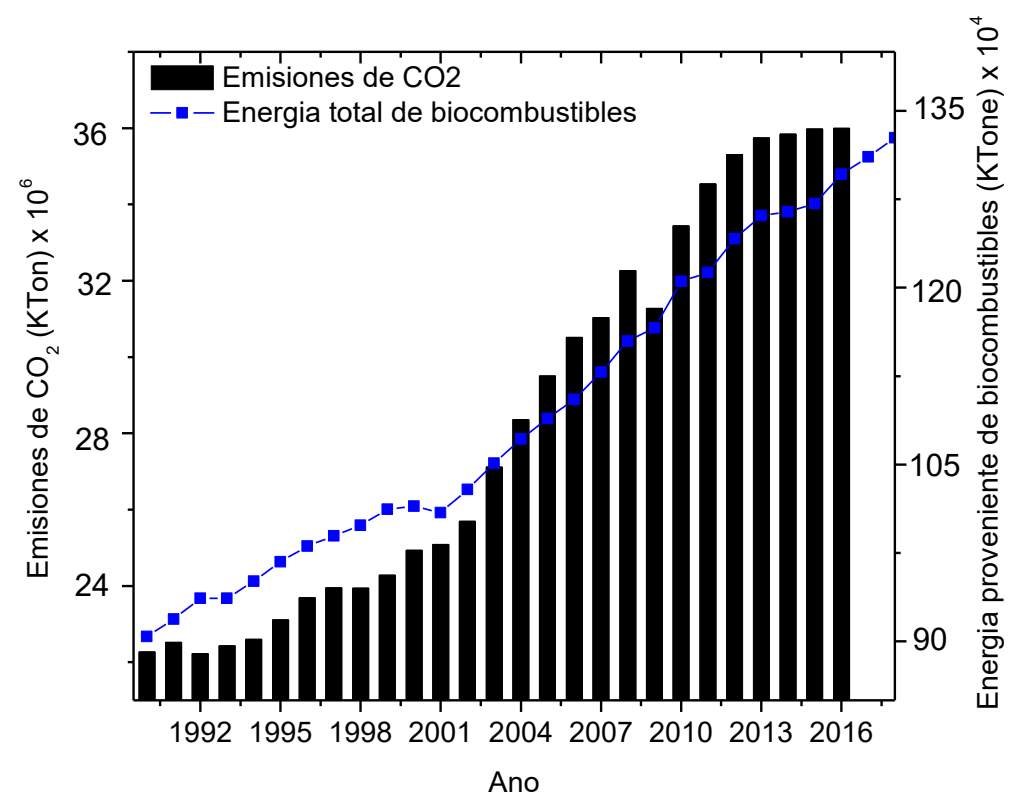

Figura 1. Emisiones de $\mathrm{CO}_{2}$ [3] y energía proveniente de biocombustibles [4] entre 1990 y 2018.

Los biocombustibles (biodiesel, bioetanol, biogás, bihidrógeno, biosyngas y biocarbón), en comparación con otras formas de energía como la energía solar y la eólica, que dependen de factores ambientales para su uso, son ventajosos ya que están fácilmente disponibles a partir de la transformación de biomasa [5], así como su naturaleza biodegradable, no tóxica y libre de azufre. Con la tendencia mostrada en la energía proveniente de los combustibles fósiles, Figura 1, se espera que para el 2040 se llegue a los 1713840 kton, convirtiéndose en una alternativa para enfrentar el desafío energético futuro y mitigar el cambio climático. Entre los diferentes biocombustibles, el biogás o biometano es una forma de energía limpia y eficiente producida a través de la digestión anaeróbica de desechos derivados de aguas residuales, vertederos, desperdicios de alimentos y materia orgánica [6]. Se ha reportado que las emisiones de gases de efecto invernadero relacionadas con la producción de biometano, en plantas de fermentación, equivalen a 44,6 $\mathrm{g} \mathrm{CO}_{2} \mathrm{eq} / \mathrm{kWh}$ [7], lo que corresponde a una reducción global de las emisiones de dióxido de carbono del $82 \%$ en comparación con los combustibles fósiles de características similares, como el gas natural, cifras que convierten al biometano en una opción muy interesante en el camino para promover el suministro de energía sostenible, limpia, segura y renovable [8]. El biogás está compuesto por metano (entre $35 \%$ y $40 \%$ ), dióxido de carbono (alrededor del $60 \%$ ) y otros componentes como amoníaco, sulfuro de hidrógeno, hidrógeno, oxígeno, nitrógeno y monóxido de carbono (CO) en concentraciones inferiores al $2 \%$ [9], sin embargo, para aumentar su valor calórico, disminuir problemas de corrosión, y potenciar su uso en aplicaciones domésticas e industriales, para la producción de calor, la generación y cogeneración de vapor y como combustible para vehículos, la concentración de dióxido de carbono debe disminuirse [10]. Por ejemplo, la especificación técnica en los Estados Unidos puntualiza que para su uso, el biogás requiere concentraciones de dióxido de carbono y gases inertes $\left(\mathrm{N}_{2}\right.$ y He) menores al $2 \%$ y al $4 \%$, respectivamente [11]. 
En promedio, el poder calorífico del gas natural es $35.8 \mathrm{MJ} / \mathrm{m}^{3}$ mientras que el del biogás es proporcional a su contenido de $\mathrm{CH}_{4}$ : el biogás con contenidos de metano de $30 \%$ y $75 \%$ en volumen, presenta poderes caloríficos de 15 y $30 \mathrm{MJ} / \mathrm{Nm}^{3}$, respectivamente [12]. Para su uso en aplicaciones comerciales e industriales, la concentración mínima de $\mathrm{CH}_{4}$ en el biogás debe ser del $90 \%$ [13], ya que porcentajes por encima de este valor reducen su poder calorífico, aumenta los costos de compresión y transporte y limita su viabilidad económica [14]. La presencia de dióxido de carbono en el biogás causa problemas al congelarse en las válvulas de control de flujo y los puntos de medición [15]. Por lo tanto, la purificación del biogás minimizando el contenido de $\mathrm{CO}_{2}$ a los niveles necesarios para su aplicación eficiente con fines de calefacción, generación de electricidad y como combustible en vehículos o motores [16], ha potencializado el aumento de las investigaciones enfocadas en el uso de membranas para la mejora del biogás. La Figura 2 muestra el número de publicaciones relacionadas con la separación de $\mathrm{CO}_{2} / \mathrm{CH}_{4}$ entre 2010 y 2020.

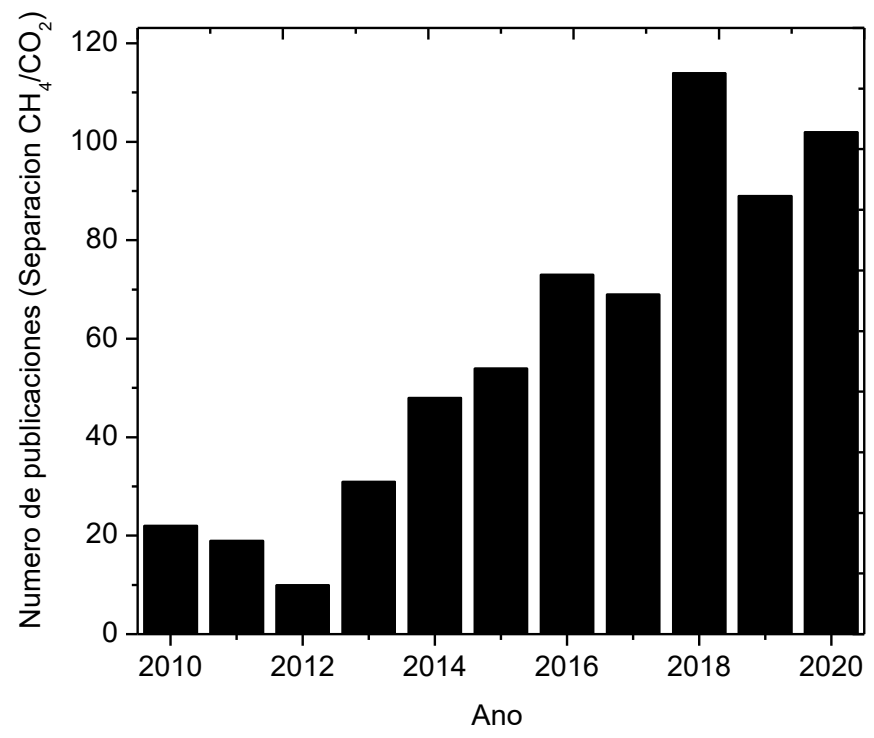

Figura 2. Número de publicaciones en separación de mezclas de $\mathrm{CO}_{2} / \mathrm{CH}_{4}$.

Como se muestra en la Figura 2, en los últimos 10 años se ha visto un rápido aumento en el número de publicaciones, relacionadas con tópicos de investigación de alternativas enfocadas en los procesos de separación de dióxido de carbono y metano, evidenciando claramente el interés por la investigación en este campo. Entre las metodologías más comunes de separación de dióxido de carbono se encuentran la destilación criogénica, la adsorción, la absorción con amina y la utilización de membranas [17]. La descripción, las ventajas y desventajas de cada una de estas metodologías se enuncian en la tabla 1.

Tabla 1. Metodologías de separación de mezclas $\mathrm{CO}_{2} / \mathrm{CH}_{4}$, descripción, ventajas y desventajas.

\begin{tabular}{|c|c|c|c|c|}
\hline $\begin{array}{l}\text { Metodología } \\
\text { de } \\
\text { separación }\end{array}$ & Descripción & Ventajas & Desventajas & Ref \\
\hline bsorción & $\begin{array}{l}\text { Reacción química con } \\
\text { solventes líquidos ióni- } \\
\text { cos modificados o ami- } \\
\text { nas. }\end{array}$ & $\begin{array}{l}\text { - Alta capacidad de ab- } \\
\text { sorción del } \mathrm{CO}_{2} \text {. } \\
\text { - Resistente a alta pre- } \\
\text { sión } \\
\text { - Alta selectividad } \\
\text { - Solventes con buena } \\
\text { capacidad de absorción }\end{array}$ & $\begin{array}{l}\text { - Altos costos energé- } \\
\text { ticos para la recupe- } \\
\text { ración de solventes. } \\
\text { - Proceso de dos pa- } \\
\text { sos regidos por el } \\
\text { equilibrio termodiná- } \\
\text { mico. } \\
\text { - Producción de com- } \\
\text { puestos volátiles }\end{array}$ & $\begin{array}{l}{[18]} \\
{[19]}\end{array}$ \\
\hline
\end{tabular}




\begin{tabular}{|c|c|c|c|}
\hline & & & $\begin{array}{l}\text { - Corrosión de equi- } \\
\text { pos. }\end{array}$ \\
\hline Adsorción & $\begin{array}{l}\text { Captura selectiva del } \\
\mathrm{CO}_{2} \text { en la superficie de } \\
\text { un adsorbente por dife- } \\
\text { rentes mecanismos quí- } \\
\text { micos (quimisorción) o fí- } \\
\text { sicos (fisisorción). }\end{array}$ & $\begin{array}{l}\text { - Eficiente a bajas con- } \\
\text { centraciones de } \mathrm{CO}_{2} \text {. } \\
\text { - Separación completa } \\
\text { de } \mathrm{CO}_{2} \text {. } \\
\text { - Alta pureza del pro- } \\
\text { ducto }\end{array}$ & $\begin{array}{l}\text { - Alto consumo de [20] } \\
\text { energía }\end{array}$ \\
\hline $\begin{array}{l}\text { Método criogé- } \\
\text { nico }\end{array}$ & $\begin{array}{l}\text { Permite la separación } \\
\text { del } \mathrm{CO}_{2} \text { en mezclas } \\
\mathrm{CO}_{2} / \mathrm{CH}_{4} \text { con base en } \\
\text { sus diferentes propieda- } \\
\text { des de condensación y } \\
\text { sublimación. }\end{array}$ & $\begin{array}{l}\text { - Obtención directa de } \\
\mathrm{CO}_{2} \text { liquido }\end{array}$ & $\begin{array}{l}\text { Elevado consumo } \\
\text { de energía para efec- } \\
\text { tuar el proceso. } \\
\text { - Bloqueo del sistema } \\
\text { de criogenización por } \\
\text { retención de agua. . }\end{array}$ \\
\hline $\begin{array}{l}\text { Uso de mem- } \\
\text { branas }\end{array}$ & $\begin{array}{l}\text { Separación física a tra- } \\
\text { vés de un barrera semi- } \\
\text { permeable }\end{array}$ & $\begin{array}{l}\text { - Tamaño reducido y } \\
\text { simplicidad operativa. } \\
\text { - Bajo costo de capital } \\
\text { - Bajo consumo de ener- } \\
\text { gía } \\
\text { - Bajo impacto ambiental }\end{array}$ & $\begin{array}{l}\text { - Pureza moderada [22] } \\
\text { del producto. } \\
\text { - Proceso no maduro }\end{array}$ \\
\hline
\end{tabular}

Tal como se muestra en la Tabla 1, entre las metodologías de separación de dióxido de carbono el uso de membranas tiene un gran potencial dado que esta puede ser utilizada como una estrategia de bajo impacto ambiental, con bajo consumo de energía y simplicidad en el proceso, además con requisitos de espacio reducidos, de fácil operación y mantenimiento [23]. El uso de membranas puede evitar todos los inconvenientes derivados de la absorción en disolventes o la adsorción en sólidos como son la corrosión, la degradación y los altos requerimientos energéticos. La separación de mezclas de dióxido de carbono y metano, utilizando tecnología de membranas, depende de factores tales como: 1) la permeabilidad al $\mathrm{CO}_{2}$; 2) la selectividad $\mathrm{CO}_{2} / \mathrm{CH}_{4}$; 3) la estabilidad del material; 4) la resiliencia a los problemas de envejecimiento y plastificación; y 5) la sostenibilidad del costo de fabricación a nivel industrial [24]. Generalmente, la atención, se centra particularmente en la productividad (permeabilidad del dióxido de carbono) y la eficiencia (selectividad $\mathrm{CO}_{2} / \mathrm{CH}_{4}$ ). Para lograr una alta permeabilidad y selectividad, se han estudiado varios materiales de membrana que de acuerdo con su naturaleza pueden clasificarse en membranas poliméricas, membranas inorgánicas y membranas de matriz mixta [25].

Las membranas poliméricas muestran alta flexibilidad y ductilidad, buena capacidad de formación de película y procesabilidad, pero generalmente están limitadas por un equilibrio entre la permeabilidad y la selectividad [26], y aunque se ha demostrado una mejora en este tipo de membranas, todavía no se ha logrado el equilibrio entre estas dos propiedades [27]. De otro lado, las membranas inorgánicas son superiores en términos de selectividad y flux de gases, además de presentar alta resistencia térmica, química y mecánica, y porosidad uniforme, pero su baja capacidad de procesamiento, fragilidad y un mayor costo de capital limita su aplicación comercial [28]. Las membranas mixtas, llamadas comúnmente MMM (mixed matrix membrane, de sus iniciales en inglés) están compuestas por un polímero con relleno inorgánico, combinan la permeabilidad del material polimérico y la selectividad de material inorgánico para crear una membrana híbrida [29].

Dada la creciente necesidad de enriquecer la cantidad de metano y aumentar el poder calorífico del biogás en aras de garantizar su comerciabilidad, la separación de $\mathrm{CO}_{2}$ de las mezclas de $\mathrm{CO}_{2} / \mathrm{CH}_{4}$ mediante la tecnología de membranas se convierte en una aplicación prometedora en cuanto a su notable ahorro de energía y su menor huella de carbono en comparación con los procesos tradicionales de separación. En este trabajo se realiza una revisión del estado de arte de trabajos publicados entre 2010 y 2020 , sobre el uso de membranas de naturaleza polimérica, inorgánica y de matriz mixta en la separación de mezclas de $\mathrm{CH}_{4} / \mathrm{CO}_{2}$. El propósito de esta revisión es destacar y discutir los avances recientes y las perspectivas futuras relacionadas con el uso de estos materiales.

\section{DEFINICION, CLASIFICACION Y MECANISMOS DE SEPARACION CON MEMBRANAS}


Una membrana es una barrera física que permite el transporte diferencial de componentes; la fracción rechazada por la membrana se llama "retenido" y la que la atraviesa se llama "permeado [30]. Las membranas se utilizan en la permeación de mezclas gaseosas [31], pervaporación [32], ultrafiltración [33] y ósmosis inversa [34], procesos ampliamente utilizados en la industria de alimentos, de petróleos, farmacéutica y en el tratamiento de efluentes gaseosos y líquidos [35]. La clasificación y mecanismos de transporte a través de las membranas se muestran en la Figura 3.

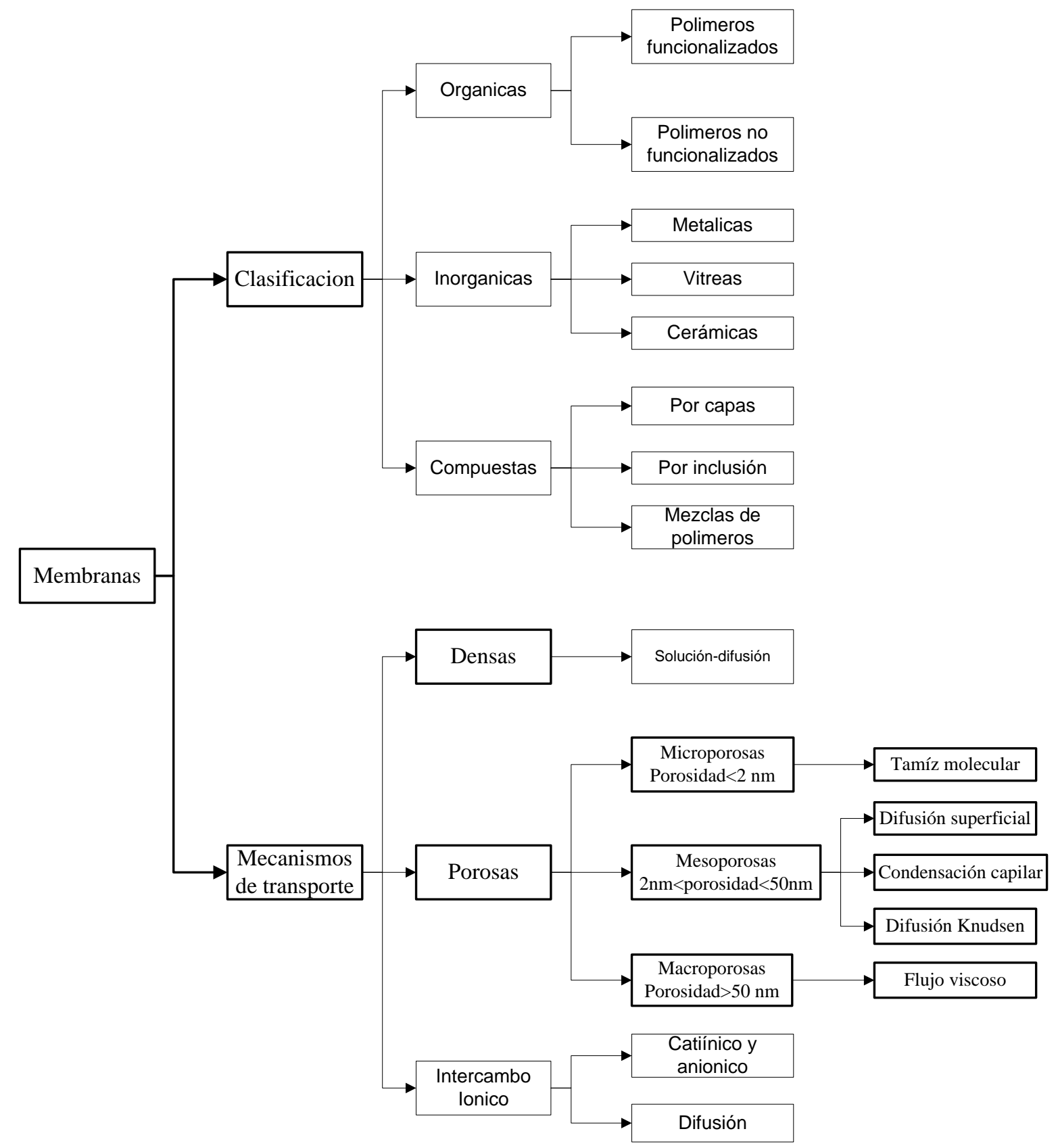

Figura 3. Clasificación y mecanismos de transporte de masa a través de membranas

En la actualidad, las membranas poliméricas dominan el mercado de membranas debido a los bajos costos de sus precursores y la facilidad de fabricación a gran escala. En principio, el mecanismo de las membranas poliméricas en la separación de gases está dado principalmente por un proceso de solucióndifusión. Las membranas poliméricas empleadas para la separación de mezclas de $\mathrm{CH}_{4} / \mathrm{CO}_{2}$ son preparadas a partir de acetato de celulosa, poliamida, polímeros aromáticos con anillos heterocíclicos y polímeros de microporosidad intrínseca [36]. Para la purificación de gas natural, la plastificación es de gran 
preocupación debido a que da como resultado una baja selectividad de las membranas. Las cadenas de polímeros se plastifican por efecto de las altas concentraciones de dióxido de carbono de modo que la permeabilidad del $\mathrm{CH}_{4}$ se acelera y la selectividad se deteriora. Para resolver este problema, se han desarrollado varios métodos, entre los cuales la reticulación (realizada por tratamientos térmicos, químicos y con luz ultravioleta) es reconocida como uno de los enfoques más efectivos al inhibir la hinchazón y la movilidad segmentaria [37].

Las membranas inorgánicas presentan mayor estabilidad en condiciones extremas de temperatura y presión, y rendimientos más altos que las membranas orgánicas. En general, las membranas inorgánicas se pueden dividir en dos categorías: de tipo poroso y no poroso. Las membranas inorgánicas porosas incluyen típicamente membranas de zeolita, las cuales contienen poros de tamaño nanométrico [38]. El crecimiento in situ y el crecimiento secundario, sobre la superficie de un soporte, son las estrategias mayoritariamente empleadas para la elaboración de este tipo de membranas. Sin embargo, dado que la nucleación y el crecimiento ocurren al mismo tiempo, la membrana resultante es propensa a tener defectos por el método de crecimiento in situ. Por el contrario, el crecimiento secundario separa estos dos pasos para optimizar la microestructura, en este, las semillas cristalinas se sintetizan y posteriormente se depositan sobre el sustrato mediante varios enfoques, como recubrimiento por inmersión, por rotación y por frotamiento. Posteriormente, las semillas crecen in situ para formar una membrana sin defectos. No obstante, la fabricación escalable de membranas inorgánicas para la separación de gases sigue siendo un desafío, lo que constituye un gran obstáculo para sus aplicaciones prácticas [39]. Las membranas inorgánicas no porosas se refieren a compuestos de dos fases que contienen cerámica de óxido de conducción mixta y fases de carbonato fundido [40]. Las membranas inorgánicas no porosas suelen tener una selectividad mayor de $\mathrm{CO}_{2} / \mathrm{CH}_{4}$ pero una baja permeabilidad de $\mathrm{CO}_{2}$, lo que no resulta atractivo para los requisitos prácticos. Por lo tanto, nuestra atención se centrará principalmente en las membranas inorgánicas porosas.

Limitadas a la relación de compensación entre permeabilidad y selectividad, las membranas de matriz mixta han atraído una gran atención y surgieron como un enfoque prometedor para resolver este problema. Al introducir nanomateriales con estructuras finamente controladas en la matriz polimérica, las membranas de matriz mixta integran la procesabilidad de los polímeros con excelentes propiedades de separación de las cargas [41]. A la fecha, se han reportado varios nanomateriales como relleno en las membranas de matriz mixta, tales como: 1) nanotubos de carbono, los cuales aumentan la permeabilidad del dióxido de carbono y la selectividad de la membrana [42], 2) óxido de grafeno, el cual permite la interacción del $\mathrm{CO}_{2}$ con el oxígeno contenido en su estructura, mejorando la separación de $\mathrm{CO}_{2} / \mathrm{CH}_{4}$ [43] y 3 ) zeolitas, las cuales su modifican su estructura superficial mejorando su compatibilidad con la matriz orgánica, reflejada ésta en un aumento en la selectividad hacia el $\mathrm{CO}_{2}$ [44].

Tal como se muestran en la Figura 3, existen diferentes tipos de mecanismos de transporte a través de las membranas, los cuales se describen brevemente a continuación:

\section{Solución-Difusión}

Se considera que este mecanismo ocurre en cinco etapas: (1) transporte de un componente desde la corriente de entrada a la superficie de la membrana, (2) distribución del componente entre el líquido y la membrana, (3) transporte a través de la membrana, (4) desorción del componente en fase vapor en el lado del permeado y (5) transporte desde esta otra superficie de la membrana al seno de la fase vapor. El transporte a través de la membrana depende del coeficiente de permeabilidad, el coeficiente de difusión y el coeficiente de solubilidad [45]

Tamiz molecular

Mecanismo altamente selectivo, que ocurre cuando el tamaño de poro de la membrana es muy pequeño (del orden del diámetro cinético de las especies de interés); la membrana solo permite el paso de las moléculas con un diámetro cinético menor que el diámetro de poro [46].

Difusión superficial

Tiene lugar cuando uno de los componentes a separar se adsorbe de forma preferencial en los poros de un material inorgánico, dándose posteriormente la difusión a través de los sitios de adsorción. Puesto que las moléculas de la especie adsorbida tienden a bloquear total o parcialmente la entrada de otras moléculas a los poros, es un mecanismo parcialmente selectivo [47].

Condensación capilar 
Este mecanismo se presenta en mezclas de gases con vapores condensables. Cuando la presión a través de la membrana es menor a la presión de saturación del vapor, se da la condensación preferencial del componente que está en mayor concentración. El componente condensado en los poros es evaporado a baja presión en el otro lado de la membrana, alcanzándose alta selectividad [48].

Flujo Knudsen

Cuando el tamaño de los poros es menor que la trayectoria libre media de las moléculas [49], estimada a partir de la teoría cinética de los gases, la frecuencia de colisiones molécula - pared es mayor a la de colisiones molécula - molécula, y la permeación es inversamente proporcional a la raíz cuadrada del peso molecular del gas teniendo menor flux los componentes más pesados. Además, la permeación es directamente proporcional a la temperatura e independiente de la presión [50].

Flujo viscoso

Es un mecanismo de transporte no selectivo observado en membranas porosas. En concreto, el choque entre las moléculas es más frecuente que su choque contra la pared de la membrana, dando como resultado que la permeación a través de la membrana esté determinada por la viscosidad de los gases, teniendo menor flux los componentes más viscosos. En este mecanismo la permeación aumenta con la presión y disminuye con la temperatura [51].

Intercambio iónico

En este tipo de mecanismo se permite el paso selectivo de iones con carga opuesta (contraiones), mientras que obstruyen iones con carga similar (co-iones), la fracción de corriente transportada en relación con la corriente total se define como el número de transporte [27].

Dada la importancia del tipo de material de la membrana y el mecanismo de transporte a través de la misma, en la siguiente sección se describen las características y resultados más relevantes de las membranas de naturaleza polimérica, inorgánica e hibrida en la separación de mezclas de dióxido de carbono y metano.

\section{SEPARACION DE MEZCLAS DE $\mathrm{CH}_{4} / \mathrm{CO}_{2}$ MEDIANTE MEMBRANAS POLIMERICAS}

La Tabla 2 muestra las condiciones experimentales, la permeabilidad y factor de separación de membranas poliméricas empleadas en la separación de mezclas de dióxido de carbono y metano.

Tabla 2. Condiciones experimentales, permeabilidad y factor de separación de membranas poliméricas.

\begin{tabular}{|c|c|c|c|c|c|c|c|}
\hline Ent. & $\begin{array}{c}\text { Material } \\
\text { membrana }\end{array}$ & $\begin{array}{c}\mathrm{CO}_{2} / \mathrm{CH}_{4} \\
\text { gas entrada }\end{array}$ & $\begin{array}{c}\text { Presión } \\
\text { (bar) }\end{array}$ & $\begin{array}{l}\text { Temp } \\
\text {. (K) }\end{array}$ & $\begin{array}{c}\mathrm{PCO}_{2} \\
\text { (ba- } \\
\text { rrer) } \\
\end{array}$ & $\begin{array}{l}\text { Factor de } \\
\text { separación } \\
\mathrm{CO}_{2} / \mathrm{CH}_{4}\end{array}$ & Ref \\
\hline 1 & 6FDA-TMPDA & $10 \%$ (vol.) & 10 & 308 & 605 & 13.2 & [52] \\
\hline 2 & PIM & 10\% (vol.) & 10 & 308 & 5613 & 15.8 & \\
\hline 3 & Teflón AF1600 & $10 \%$ (vol.) & 10 & 308 & 527 & 13.0 & \\
\hline 4 & 6FDA-TMPDA-48 a & 10\% (vol.) & 10 & 308 & 640 & 13.5 & \\
\hline 5 & 6FDA-TMPDA-50 a & $10 \%$ (vol.) & 10 & 308 & 617 & 12.8 & \\
\hline 6 & 6FDA-TMPDA-54 a & $10 \%$ (vol.) & 10 & 308 & 607 & 12.8 & \\
\hline 7 & PIM--40 a & $10 \%$ (vol.) & 10 & 308 & 2547 & 14.1 & \\
\hline 8 & PIM-43 a & $10 \%$ (vol.) & 10 & 308 & 2007 & 14.0 & \\
\hline 9 & PIM-45 a & $10 \%$ (vol.) & 10 & 308 & 1803 & 13.8 & \\
\hline 10 & CHFM-80C ${ }^{b}$ & $50 \%(\mathrm{~mol})$ & 10 & 333 & 550 & 55.0 & [53] \\
\hline 11 & CHFM-80C & $50 \%(\mathrm{~mol})$ & 20 & 333 & 480 & 43.0 & \\
\hline 12 & CHFM-80C & $50 \%(\mathrm{~mol})$ & 30 & 333 & 400 & 40.0 & \\
\hline 13 & CHFM-80C & $50 \%(\mathrm{~mol})$ & 40 & 333 & 375 & 31.0 & \\
\hline 14 & CHFM-80C & $50 \%(\mathrm{~mol})$ & 50 & 333 & 370 & 30.0 & \\
\hline 15 & CHFM-140C & $50 \%(\mathrm{~mol})$ & 10 & 333 & 177 & 175 & \\
\hline
\end{tabular}




\begin{tabular}{|c|c|c|c|c|c|c|c|}
\hline 16 & CHFM-140C & $50 \%$ (mol) & 20 & 333 & 160 & 150 & \\
\hline 17 & CHFM-140C & $50 \%(\mathrm{~mol})$ & 30 & 333 & 148 & 140 & \\
\hline 18 & CHFM-140C & $50 \%$ (mol) & 40 & 333 & 140 & 131 & \\
\hline 19 & CHFM-140C & $50 \%(\mathrm{~mol})$ & 50 & 333 & 127 & 129 & \\
\hline 20 & Pebax 1657 & $50 \%(\mathrm{~mol})$ & 4 & 293 & $30^{*}$ & 32 & [54] \\
\hline 21 & I-Pebax 1657 & $50 \%(\mathrm{~mol})$ & 4 & 293 & $35^{\star}$ & 34 & \\
\hline 22 & 6FDA-ODA-OHc & $10 \%$ (vol.) & 10.3 & 308 & 18.6 & 48.9 & [55] \\
\hline 23 & 6FDA-ODA-1H & $10 \%$ (vol.) & 10.3 & 308 & 15.9 & 38.9 & \\
\hline 24 & 6FDA-ODA-2H & $10 \%$ (vol.) & 10.3 & 308 & 15.6 & 46.8 & \\
\hline 25 & 6FDA-ODA-3H & $10 \%$ (vol.) & 10.3 & 308 & 15.1 & 49.7 & \\
\hline 26 & 6FDA-ODA-4H & 10\% (vol.) & 10.3 & 308 & 15.7 & 58.1 & \\
\hline 27 & 6FDA-ODA-5H & $10 \%$ (vol.) & 10.3 & 308 & 12.9 & 62.0 & \\
\hline 28 & 6FDA-ODA-24H & $10 \%$ (vol.) & 10.3 & 308 & 16.6 & 49.6 & \\
\hline 29 & 6F-PAl-1 & Gas puro & 4.5 & 308 & 32.8 & n.r. & [56] \\
\hline 30 & 6F-PAI-2 & Gas puro & 4.5 & 308 & 14.2 & n.r. & \\
\hline 31 & $6 F-P A-3$ & Gas puro & 4.5 & 308 & 21.6 & n.r. & \\
\hline 32 & Torlon & Gas puro & 4.5 & 308 & 0.7 & n.r. & \\
\hline 33 & PSF-DEG & Gas puro & 2 & 308 & 84.97 & n.r. & [57] \\
\hline 34 & PSF-DEG & Gas puro & 3 & 308 & 45.35 & n.r. & \\
\hline 35 & PSF-DEG & Gas puro & 4 & 308 & 34.92 & n.r. & \\
\hline 36 & PSF-DEG & Gas puro & 5 & 308 & 29.08 & n.r. & \\
\hline 37 & PSF-DEG & Gas puro & 5.8 & 308 & 24.57 & n.r. & \\
\hline 38 & PIM-PI:1-4d & $50 \%(\mathrm{~mol})$ & 2 & 303 & 3011 & 17.0 & [58] \\
\hline 39 & PIM-PI:1-6 d & $50 \%(\mathrm{~mol})$ & 2 & 303 & 1660 & 17.7 & \\
\hline 40 & PIM-PI:1-8d & $50 \%(\mathrm{~mol})$ & 2 & 303 & 1435 & 17.9 & \\
\hline
\end{tabular}

aEspesor de la membrana (6FDA-TMPDA-48: espesor de $48 \mu \mathrm{m}$ ), 'bTemperatura de síntesis (CHFM-80C: Síntesis a $\left.80^{\circ} \mathrm{C}\right)$. ${ }^{\mathrm{C}}$ Tiempo de síntesis (6FDA-ODA-1H: síntesis por $1 \mathrm{~h}$ ). ${ }^{\mathrm{d}}$ relación PIM-PI n.r.: no reportada. Pco2: Permeabilidad del $\mathrm{CO}_{2 .}{ }^{* \star}$ Permeancia (GPU).

6FDA-TMPDA: polyimide 4,4-(hexafluoroisopropylidene) diphthalic anhydride 2,3,5,6-tetramethyl1,4-phenylenediamine. PIM: Polymer of Intrinsic Micro-porosity. CHFM: Carbon hollow fiber membranes. I-Pebax 1657: Ionic liquid modified Pebax 1657. 6FDA-ODA: Homopolyimide. 6F-PAI polyamide- imides. PSF-DEG: Polysulfone-Diethylene Glycol.

\section{SEPARACION DE MEZCLAS $\mathrm{CH}_{4} / \mathrm{CO}_{2}$ MEDIANTE MEMBRANAS INORGANICAS}

La Tabla 3 muestra las condiciones experimentales, la permeabilidad y factor de separación de membranas inorgánicas empleadas en la separación de mezclas de dióxido de carbono y metano.

Tabla 3. Condiciones experimentales, permeabilidad y factor de separación de membranas inorgánicas.

\begin{tabular}{|c|c|c|c|c|c|c|c|}
\hline Ent. & $\begin{array}{l}\text { Material mem- } \\
\text { brana }\end{array}$ & $\begin{array}{c}\mathrm{CO}_{2} / \mathrm{CH}_{4} \\
\text { gas entrada }\end{array}$ & $\begin{array}{l}\text { Presión } \\
\text { (bar) }\end{array}$ & $\begin{array}{l}\text { Temp } \\
\text { (K) }\end{array}$ & $\begin{array}{c}\mathrm{PCO}_{2} \\
\text { (ba- } \\
\text { rrer) }\end{array}$ & $\begin{array}{c}\text { Factor de } \\
\text { separa- } \\
\text { ción } \mathrm{CO}_{2} \\
/ \mathrm{CH}_{4}\end{array}$ & Ref. \\
\hline 1 & SSZ-13 & $50 \%$ (vol.) & 0.2 & 298 & $1.19^{*}$ & 305 & [59] \\
\hline 2 & SSZ-13 & $50 \%$ (vol.) & 0.2 & 298 & 1.21 & 183 & \\
\hline 3 & SSZ-13 & $50 \%$ (vol.) & 0.2 & 298 & 1.08 & 152 & \\
\hline 4 & AIPO-H6a & $50 \%(\mathrm{~mol})$ & 1 & 423 & $3.60^{*}$ & 91.5 & {$[60]$} \\
\hline 5 & AIPO-H12 & $50 \%(\mathrm{~mol})$ & 1 & 423 & 3.75 & 81.4 & \\
\hline 6 & AIPO-H48 & $50 \%(\mathrm{~mol})$ & 1 & 423 & 3.34 & 89.0 & \\
\hline 7 & AIPO-H48 & $50 \%(\mathrm{~mol})$ & 2 & 298 & 3.60 & n.r. & \\
\hline 8 & AIPO-H48 & $50 \%(\mathrm{~mol})$ & 2 & 323 & 2.70 & n.r. & \\
\hline 9 & AlPO-H48 & $50 \%(\mathrm{~mol})$ & 2 & 348 & 2.00 & n.r. & \\
\hline 10 & AIPO-H48 & $50 \%(\mathrm{~mol})$ & 2 & 373 & 1.60 & n.r. & \\
\hline 11 & AIPO-H48 & $50 \%(\mathrm{~mol})$ & 2 & 398 & 1.25 & n.r. & \\
\hline 12 & AIPO-H48 & $50 \%(\mathrm{~mol})$ & 2 & 423 & 1.10 & n.r. & \\
\hline 13 & Chabazite & $50 \%(\mathrm{~mol})$ & 1 & 303 & $8.0^{*}$ & 14.0 & [61] \\
\hline
\end{tabular}




\begin{tabular}{llllllll}
14 & Chabazite & $50 \%(\mathrm{~mol})$ & 2 & 303 & 6.5 & 12.0 & \\
15 & Chabazite & $50 \%(\mathrm{~mol})$ & 3 & 303 & 5.4 & 10.0 & \\
16 & Chabazite & $50 \%(\mathrm{~mol})$ & 4 & 303 & 5.0 & 8.0 & \\
17 & Chabazite & $50 \%(\mathrm{~mol})$ & 5 & 473 & 4.8 & 7.5 & \\
\hline 18 & Si-CHA-26b & $50 \%(\mathrm{~mol})$ & 7 & 298 & $92^{*}$ & 38 & {$[62]$} \\
19 & Si-CHA-47 & $50 \%(\mathrm{~mol})$ & 7 & 298 & 90 & 58 & \\
20 & Si-CHA-152 & $50 \%(\mathrm{~mol})$ & 7 & 298 & 65 & 45 & \\
\hline 21 & ZSM-58-24c & $50 \%(\mathrm{~mol})$ & 11 & 298 & $9.2^{*}$ & 87 & {$[63]$} \\
22 & ZSM-58-36 & $50 \%(\mathrm{~mol})$ & 11 & 298 & 5.6 & 77 & \\
23 & ZSM-58-48 & $50 \%(\mathrm{~mol})$ & 11 & 298 & 3.0 & 9.8 & \\
24 & ZSM-58-72 & $50 \%(\mathrm{~mol})$ & 11 & 298 & 0.04 & 1.4 & \\
\hline 25 & LTA-Zeolite & $50 \%(\mathrm{~mol})$ & 1 & 303 & $3.0^{*}$ & 17.5 & {$[64]$} \\
26 & LTA-Zeolite & $50 \%(\mathrm{~mol})$ & 2 & 303 & 3.2 & 20.0 & \\
27 & LTA-Zeolite & $50 \%(\mathrm{~mol})$ & 3 & 303 & 3.6 & 22.0 & \\
\hline 28 & T-Zeolite & $50 \%(\mathrm{~mol})$ & 1 & 308 & n.r. & 6.0 & {$[65]$} \\
29 & T-Zeolite & $50 \%(\mathrm{~mol})$ & 3 & 308 & n.r. & 3.8 & \\
30 & T-Zeolite & $50 \%(\mathrm{~mol})$ & 5 & 308 & n.r. & 3.1 & \\
31 & T-Zeolite & $50 \%(\mathrm{~mol})$ & 7 & 308 & n.r. & 2.6 & \\
\hline 32 & 5 A-Zeolite & $40 \%(\mathrm{~mol})$ & 0.74 & 303 & $1.65^{*}$ & 81 & {$[66]$} \\
33 & 5 A-Zeolite & $40 \%(\mathrm{~mol})$ & 1.20 & 303 & 2.11 & 56 & \\
34 & 5A-Zeolite & $40 \%(\mathrm{~mol})$ & 1.70 & 303 & 2.49 & 85 & \\
35 & 5A-Zeolite & $40 \%(\mathrm{~mol})$ & 2.29 & 303 & 2.59 & 47 & \\
36 & 5 A-Zeolite & $40 \%(\mathrm{~mol})$ & 3.21 & 303 & 2.84 & 44 & \\
37 & 5A-Zeolite & $40 \%(\mathrm{~mol})$ & 4.35 & 303 & 2.71 & 21 & \\
\hline
\end{tabular}

a Tiempo de síntesis. AIPO-H6 (membrana sintetizada por seis horas, ${ }^{c}$ Tiempo de síntesis. M-58-36 (síntesis durante 36 horas). ${ }^{*} \mathrm{~mol} \cdot\left(\mathrm{m}^{2} \cdot \mathrm{s} \cdot \mathrm{Pa}\right)^{-1}{ }^{* *}$ flujo $(\mathrm{mol} / \mathrm{Kg})$

SSZ-13: Zeolite, AIPO: aluminophosphates. Si-CHA: Silice-Zeolite. Si-CHA: ZSM-58: all sílica

\section{SEPARACION DE MEZCLAS $\mathrm{CH}_{4} / \mathrm{CO}_{2}$ MEDIANTE MEMBRANAS DE MATRIZ MIXTA}

La Tabla 4 muestra las condiciones experimentales, la permeabilidad y el factor de separación de membranas de matriz mixta empleadas en la separación de mezclas $\mathrm{CO}_{2} / \mathrm{CH}_{4}$.

Tabla 4. Condiciones experimentales, permeabilidad y factor de separación de membranas mixtas.

\begin{tabular}{cccccccc}
\hline Ent. & $\begin{array}{c}\text { Material } \\
\text { membrana }\end{array}$ & $\begin{array}{c}\mathrm{CO}_{2} / \mathrm{CH}_{4} \\
\text { gas entrada }\end{array}$ & $\begin{array}{c}\text { Presión } \\
(\text { bar })\end{array}$ & $\begin{array}{c}\text { Temp. } \\
\mathrm{K})\end{array}$ & $\begin{array}{c}\mathrm{PCO}_{2} \\
\text { (barrer) }\end{array}$ & $\begin{array}{c}\text { Factor de } \\
\text { separación } \\
\mathrm{CO}_{2} / \mathrm{CH}_{4}\end{array}$ & $\begin{array}{c}\text { Ref } \\
.\end{array}$ \\
\hline 1 & FDA-DAM:DABA & $50 \%(\mathrm{~mol})$ & 2 & 303 & 199 & 35.9 & {$[67]$} \\
2 & FDA-DAM:DABA-5 & $50 \%(\mathrm{~mol})$ & 2 & 303 & 360 & 34.8 & \\
3 & FDA-DAM:DABA-10 & $50 \%(\mathrm{~mol})$ & 2 & 303 & 340 & 28.6 & \\
4 & FDA-DAM:DABA-15 & $50 \%(\mathrm{~mol})$ & 2 & 303 & 315 & 16.2 & \\
\hline 5 & FDA-DAM:DABA & $50 \%(\mathrm{~mol})$ & 1 & 298 & 134 & 38.3 & {$[68]$} \\
6 & FDA-DAM:DABA & $50 \%(\mathrm{~mol})$ & 1 & 298 & 202 & 36.2 & \\
7 & FDA-DAM:DABA & $50 \%(\mathrm{~mol})$ & 1 & 298 & 365 & 33.0 & \\
\hline 8 & M-ZSM25-0 & $50 \%(\mathrm{~mol})$ & 3 & 308 & 10.0 & 45.0 & {$[69]$} \\
9 & M-ZSM25-5 & $50 \%(\mathrm{~mol})$ & 3 & 308 & 13.1 & 34.0 & \\
10 & M-ZSM25-8 & $50 \%(\mathrm{~mol})$ & 3 & 308 & 16.3 & 22.0 & \\
11 & M-ZSM25-10 & $50 \%(\mathrm{~mol})$ & 3 & 308 & 17.2 & 24.0 & \\
12 & M-ZSM25-15 & $50 \%(\mathrm{~mol})$ & 3 & 308 & 20.5 & 4.90 & \\
\hline 13 & Matrimid & $50 \%(\mathrm{~mol})$ & 1 & 298 & $10.0^{*}$ & 4.5 & {$[70]$} \\
14 & Matrimid/GO 5\%d & $50 \%(\mathrm{~mol})$ & 1 & 298 & $8.50^{*}$ & 6.3 & \\
15 & Matrimid/GO 10\% & $50 \%(\mathrm{~mol})$ & 1 & 298 & $7.60^{*}$ & 7 & \\
16 & Matrimid/NiDOBC 5\% & $50 \%(\mathrm{~mol})$ & 1 & 298 & $9.20^{*}$ & 5 & \\
17 & Matrimid/NiDOBC 10\% & $50 \%(\mathrm{~mol})$ & 1 & 298 & $7.50^{*}$ & 5.1 &
\end{tabular}




\begin{tabular}{|c|c|c|c|c|c|c|c|}
\hline 18 & Matrimide/comp-5\% & $50 \%(\mathrm{~mol})$ & 1 & 298 & $9.50^{*}$ & 5.3 & \\
\hline 19 & Matrimide/comp-10\% & $50 \%(\mathrm{~mol})$ & 1 & 298 & $10.2^{*}$ & 8.5 & \\
\hline 20 & Matrimide/comp-15\% & $50 \%(\mathrm{~mol})$ & 1 & 298 & $10.5^{*}$ & 10 & \\
\hline 21 & Matrimide/comp-20\% & $50 \%(\mathrm{~mol})$ & 1 & 298 & $9.80^{*}$ & 11 & \\
\hline 22 & Pebax & $50 \%(\mathrm{~mol})$ & 3 & 298 & 123 & 21.21 & [71] \\
\hline 23 & Pebax-y-Al ${ }_{2} \mathrm{O}_{3} 2 \%{ }^{\mathrm{e}}$ & $50 \%(\mathrm{~mol})$ & 3 & 298 & 125 & 21.49 & \\
\hline 24 & Pebax- $y-\mathrm{Al}_{2} \mathrm{O}_{3} 4 \%$ & $50 \%(\mathrm{~mol})$ & 3 & 298 & 129 & 21.73 & \\
\hline 25 & Pebax- $y-\mathrm{Al}_{2} \mathrm{O}_{3} 6 \%$ & $50 \%(\mathrm{~mol})$ & 3 & 298 & 139 & 22.51 & \\
\hline 26 & Pebax-- $-\mathrm{Al}_{2} \mathrm{O}_{3} 8 \%$ & $50 \%(\mathrm{~mol})$ & 3 & 298 & 159 & 24.73 & \\
\hline 27 & PVC-POEM/ZIF & $50 \%(\mathrm{~mol})$ & 3 & 298 & $4.10^{*}$ & 21.8 & [72] \\
\hline 28 & PVC-POEM/ZIF-10 & $50 \%(\mathrm{~mol})$ & 3 & 298 & $8.90^{*}$ & 14.4 & \\
\hline 29 & PVC-POEM/ZIF-30 & $50 \%(\mathrm{~mol})$ & 3 & 298 & $10.6^{*}$ & 13.3 & \\
\hline 30 & PVC-POEM/ZIF-50 & $50 \%(\mathrm{~mol})$ & 3 & 298 & $12.2^{*}$ & 11.6 & \\
\hline 31 & ZIF-8/P84-09 & $50 \%(\mathrm{~mol})$ & 3 & 298 & 2.66 & 54.1 & [73] \\
\hline 32 & ZIF-8/P84-8 & $50 \%(\mathrm{~mol})$ & 3 & 298 & 3.15 & 63.5 & \\
\hline 33 & ZIF-8/P84-17 & $50 \%(\mathrm{~mol})$ & 3 & 298 & 6.33 & 93.6 & \\
\hline 34 & ZIF-8/P84-27 & $50 \%(\mathrm{~mol})$ & 3 & 298 & 11.0 & 92.3 & \\
\hline 35 & ZIF-8/P84-31 & $50 \%(\mathrm{~mol})$ & 3 & 298 & 20.0 & 45.8 & \\
\hline 36 & Matrimid & $50 \%(\mathrm{~mol})$ & 2 & 308 & $8.34^{* *}$ & 36.3 & [74] \\
\hline 37 & Matrimid/NaY-5\% & $50 \%(\mathrm{~mol})$ & 2 & 308 & $12.2^{* *}$ & 38.9 & \\
\hline 38 & Matrimid/NaY-10\% & $50 \%(\mathrm{~mol})$ & 2 & 308 & $15.0^{* *}$ & 39.8 & \\
\hline 39 & Matrimid/NaY-15\% & $50 \%(\mathrm{~mol})$ & 2 & 308 & $17.5^{* *}$ & 43.3 & \\
\hline 40 & Matrimid/NaY-20\% & $50 \%(\mathrm{~mol})$ & 2 & 308 & $22.0^{* *}$ & 27.6 & \\
\hline 41 & Matrimid & $50 \%(\mathrm{~mol})$ & 1 & 313 & 10.2 & 33.6 & [75] \\
\hline 42 & Matrimid-10 bare $5 \mathrm{~A}$ & $50 \%(\mathrm{~mol})$ & 1 & 313 & 26.7 & 31.3 & \\
\hline 43 & Matrimid-10 bare $5 \mathrm{~A}$ & $50 \%(\mathrm{~mol})$ & 1 & 313 & 31.0 & 30.8 & \\
\hline
\end{tabular}

a.\% peso de SSZ-16, b\% peso de polisulfona PSF, c\% peso de LNZ25, d\% peso de GO, NiDOBC o composite, e\% peso de $\mathrm{Al}_{2} \mathrm{O}_{3}$, $\%$ peso de $\mathrm{ZIF}$, g\% peso de PB4, h\% peso de NaY. ${ }^{*}$ Permeancia mol $\mathrm{m} / \mathrm{m} 2 \mathrm{~s}$ bar. ${ }^{* *} \mathrm{~cm}^{3}(\mathrm{STP}) / \mathrm{cm}^{3} \mathrm{cmHg}$.

FDA-DAM:DABA: diphthalic anhydride- diaminobenzene: -diaminobenzoic acid polymide. M-ZSM25: aminosilane sodium zeolite-matrimid. GO: Graphite Oxide. NiDOBC: Nickel-dihydroxy-1,4-benzenedicarboxylic acid. PVC-POEM: poly vinyl chloride-poly oxyethylene methacrylate. PB4: Polyimide P84:

Tal como se puede observar en las tablas 2, 3 y 4, las cuales evalúan el desempeño de las membranas polimericas, inorgánicas y mixtas, respectivamente, el tiempo y la temperatura de síntesis son los parámetros de mayor influencia para la preparación de la membrana, mientras que el efecto de la presión y la temperatura sobre la permeabilidad y la selectividad son los parámetros experimentales evaluados por la mayoría de los autores para el desempeño de las membranas poliméricas e inorgánicas; en las inorgánicas, el efecto del contenido de relleno adicionado es un parámetro adicional reportado.

La permeabilidad del $\mathrm{CO} 2$ es diferente en cada una de las membranas reportadas, lo cual es resultado de las diferencias en morfología, naturaleza y afinidad de cada uno de los materiales poliméricos, inorgánicos o combinados con el dióxido de carbono. De hecho, la diferencia en la permeación y el factor de separación se debe principalmente al transporte competitivo de mezclas de $\mathrm{CO}_{2} / \mathrm{CH}_{4}$, asociado con la diferencia de cada uno de los mecanismos por los cuales se difunde o transporta el dióxido de carbono a través de la membranas bajo un gradiente de concentración entre la alimentación y el lado permeado de la membrana.

Como característica particular en las membranas poliméricas e inorgánicas, se encontró que la selectividad del dióxido de carbono sobre el metano disminuye al aumentar la presión (entradas 10-20, Tabla 2 y entradas 13-17, 28-31, 32-37, Tabla 3). Para las membranas de naturaleza inorgánica, se encontró que la permeabilidad del dióxido de carbono disminuye a medida que aumenta la temperatura debido a la elevada capacidad de adsorción del $\mathrm{CO}_{2}$ en la zeolita AIPO-18 (entradas 7-12, Tabla 3). En efecto, la permeabilidad de un gas individual es la suma de la difusión de superficie y la difusión de traslación de gas. La permeación del gas adsorbido a través de los poros de la zeolita disminuye con el aumento de temperatura hasta que la permeación está dominada principalmente por la difusión por traslación del gas. También se encontró que la permeabilidad del dióxido de carbono disminuye con el tiempo de síntesis (entradas 23-27, Tabla 2) mientras que el factor de separación alcanza un máximo a las 5 horas, lo 
que indica que las reacciones de reticulación no son instantáneas y necesitan un tiempo óptimo para producir una estructura química estable.

Con respecto a las membranas de naturaleza mixta, se encuentra que el rendimiento de separación de las membranas mejora con la introducción de diferentes materiales de naturaleza inorgánica en la matriz de polímero. Así, se encontró que un aumento en la concentración de los materiales (entradas 18-20, 23-26, 28-30, 31-35, 37-40, Tabla 3) conduce a una mejora continua y notable tanto en la permeabilidad del $\mathrm{CO}_{2}$ como en los factores de separación de $\mathrm{CO}_{2} / \mathrm{CH}_{4}$, por formación de un sistema híbrido compatible compuesto por nanopartículas inorgánicas y polímeros, rompiendo el equilibrio entre permeabilidad y selectividad de las membranas poliméricas. Sin embargo, cuando la carga aumenta por encima del valor límite (más del 31\% en peso en el caso de la membrana mixta), ZIF-8/P84 MMM (entrada 35), la aglomeración severa de nanopartículas inorgánicas en la matriz P84 es inevitable, lo que provoca defectos interfaciales no selectivos y finalmente conduce a una fuerte disminución del factor de separación $\mathrm{CO}_{2} / \mathrm{CH}_{4}$, fenómeno frecuente en la fabricación de membranas de naturaleza mixta mediante el uso de métodos convencionales de mezcla de soluciones.

\section{DESAFIOS Y PERSPECTIVAS EN LA TECNOLOGIA DE MEMBRANAS}

Acorde con la revisión de literatura, todavía existen varios desafíos en el desarrollo de membranas poliméricas. Las mejoras en la resistencia a la plastificación y el rendimiento de separación consolidarán aún más el estado de la tecnología de membranas en la purificación práctica del biogás. Representadas por zeolitas, las membranas inorgánicas exhibieron un rendimiento más alto que las membranas poliméricas. Vale la pena modificar el método de preparación actual para reducir el costo mientras se logra la fabricación a gran escala. Idealmente, las membranas de naturaleza mixta combinan las ventajas de los materiales poliméricos e inorgánicos para lograr propiedades sobresalientes. En el futuro, se deben realizar más esfuerzos para eliminar los defectos de interfase y comprender el mecanismo de transporte en las membranas de matriz mixta.

\section{CONCLUSIONES}

En esta revisión se proporcionó una descripción de la tecnología basada en membranas poliméricas, inorgánicas y de matriz mixta para la eliminación de $\mathrm{CO}_{2}$ en mezclas de dióxido de carbono y metano.

Los parámetros de fabricación de las membranas (temperatura y tiempo de sintesis) y condiciones experimentales de trabajo (presión y temperatura) son los factores reportados por la mayoría de los autores como los de mayor influencia en las propiedades de la membrana.

Las membranas poliméricas exhiben factores de separación inferiores a las membranas inorgánicas, pero actualmente dominan el mercado de membranas de separación de gases ya que se pueden procesar fácilmente en diferentes configuraciones. Además, su bajo coste de fabricación es de gran interés para aplicaciones industriales.

Las membranas inorgánicas resultan ser atractivas para procesos de separación de CO2 dada su robusta estabilidad térmica, química y mecánica. De otra parte, se requieren estudios enfocados en la reproducibilidad de estos sistemas para permitir su escalado, así como para reducir los costos de fabricación.

El tipo de relleno y la matriz, la eliminación de defectos entre dos fases y la propuesta de modelos de transporte, son los principales desafíos en el desarrollo de membranas de matriz mixta.

Se espera que los esfuerzos futuros de investigación y desarrollo idealmente proporcionen membranas más duraderas con un rendimiento mejorado que se puede ampliar fácilmente para cumplir con el potencial de estas membranas en la industria de la separación de CO2.

\section{AGRADECIMIENTOS}

Los autores agradecen el apoyo a la Universidad Cooperativa de Colombia para el desarrollo de este trabajo de revisión. C. Cardona, D. Molina y R. González agradecen especialmente al Semillero en Energía y Medio Ambiente. 


\section{REFERENCIAS BIBLIOGRÁFICAS}

[1] Yang, F. Chou, J. Dong, W. Sun,M. Zhao, W. (2020). Adaption to climate change risk in eastern China: Carbon emission characteristics and analysis of reduction path, Physics and. Chemistry of the Earth. 115 102829. http://doi.org/10.1016/j.pce.2019.102829.

[2] Li, M. Luo, N. Lu, Y. (2017). Biomass energy technological paradigm (BETP): Trends in this sector, Sustainability. 9 (4) 1-28. http://doi.org/10.3390/su9040567.

[3] Banco mundial (13 de enero de 2021) Emisones de dioxido de carbono banco mundial. https://datos.bancomundial.org/indicator/EN.ATM.CO2E.KT.

[4] International energy agency (13 de enero) total energy supply by source, world 1990-2018. https://www.iea.org/data-andstatistics?country=world\&fuel=energy\%20supply\&indicatorbysource.

[5] Ullah, K. Sharma, V. Ahmad, M. Lv, P. Krahl, J. Wang,Z. et al. (2018) The insight views of advanced technologies and its application in bio-origin fuel synthesis from lignocellulose biomasses waste, a review, Renewable Sustainable Energy Review. 82 (2018) 3992-4008. http://doi.org/10.1016/j.rser.2017.10.074.

[6] Singh, D. Sharma, D. Soni, S. Sharma, S. Kumar. P. Sharma, P. halani, A. A review on feedstocks, production processes, and yield for different generations of biodiesel, (2020) Fuel. 262 116553. http://doi.org/10.1016/j.fuel.2019.116553.

[7] Adelt, M. Wolf, D.Vogel, A. (2011) LCA of biomethane, Journal of Natural Gas Science. Engineering . 3 (5) 646-650. http://doi.org/10.1016/j.jngse.2011.07.003.

[8] Kapoor, R. Ghosh, P. Tyagi, B. Vijay, V. Vijay, V Thakur, I. et al., (2020) Advances in biogas valorization and utilization systems: A comprehensive review, Journal of Cleaner Production. 273 123052. http://doi.org/10.1016/j.jclepro.2020.123052.

[9] Chowdhury, T. Chowdhury, H. Hossain, N. Ahmed, A. Hossen, M. Chowdhury, Pet al., (2020) Latest advancements on livestock waste management and biogas production: Bangladesh's $\begin{array}{lllll}\text { perspective, Journal of } & \text { Cleaner } & \text { Production. } & 272 & 122818 .\end{array}$ http://doi.org/10.1016/j.jclepro.2020.122818

[10] Puricelli, S. Cardellini, G. Casadei, S. Faedo, D. Van den Oever, A. Grosso, (M. 2020) A review on biofuels for light-duty vehicles in Europe, Renewable. Sustainable. Energy Rev. 137. 110398. http://doi.org/10.1016/j.rser.2020.110398.

[11] Li, S. Jiang, X. Sun, H. He, S. Zhang, L. Shao, L. (2019) Mesoporous dendritic fibrous nanosilica (DFNS) stimulating mix matrix membranes towards superior CO2 capture, Journal of Membrane Science. 586 185-191. http://doi.org/10.1016/j.memsci.2019.05.069.

[12] Bragança, I. Sánchez-Soberón, F. Pantuzza, G. Alves, A. Ratola, N.(2020) Impurities in biogas: Analytical strategies, occurrence, effects and removal technologies, Biomass and Bioenergy. 143. http://doi.org/10.1016/j.biombioe.2020.105878.

[13] Harasimowicz, M. Orluk, P. Zakrzewska-Trznadel, G. Chmielewski, A. (2007). Application of polyimide membranes for biogas purification and enrichment. Journal Hazard Materials. 144 (3) 698-702. http://doi.org/10.1016/j.jhazmat.2007.01.098.

[14] Medrano, J. Llosa-Tanco, M. Cechetto, V. Pacheco-Tanaka, D. Gallucci, F.(2020) Upgrading biogas with novel composite carbon molecular sieve (CCMS) membranes: Experimental and techno-economic assessment, Chemical Engineering Journal 394 (2020) 124957. http://doi.org/10.1016/j.cej.2020.124957.

[15] Al Mamun, M. Karim, M. Rahman, M. Asiri, A. Torii, S. (2016) Methane enrichment of biogas by carbon dioxide fixation with calcium hydroxide and activated carbon, Jounal of the Taiwan Institue of. Chemical Enginners . 58. 476-481. http://doi.org/10.1016/j.jtice.2015.06.029.

[16] Rafiee, A. Khalilpour, K. Prest,J. Skryabin, I. (2020) Biogas as an energy vector, Biomass and Bioenergy. 144. 105935. http://doi.org/:10.1016/j.biombioe.2020.105935.

[17] Hafeez, S. Safdar, T. Pallari, E. Manos,G. Aristodemou, E. Zhang, Z. et al., CO2 capture using membrane contactors: a systematic literature review, Frontiers of Chemical Science and engineering. 107. 1-35. http://doi.org/10.1007/s11705-020-1992-z.

[18] Fang, M. Yi, N. Di, W. Wang, T. Wang, Q. (2020) Emission and control of flue gas pollutants in CO2 chemical absorption system - A review, International Journal of Greenhouse Gas Control. 93.10294. http://doi.org/10.1016/j.jjggc.2019.102904.

[19] Xu, M. Wang, S. Xu, L. (2019) Screening of physical-chemical biphasic solvents for CO2 absorption, International Journal of Greenhouse Gas Control. 85. 199-205. http://doi.org/10.1016/j.ijggc.2019.03.015.

[20] Petrovic, B. Gorbounov, M. Masoudi Soltani, S. (2021) Influence of surface modification on 
selective $\mathrm{CO} 2$ adsorption: A technical review on mechanisms and methods, Microporous and Mesoporous Materiasl. 312. 110751. http://doi.org/10.1016/j.micromeso.2020.110751.

[21] Song, C. Liu, Q. Deng, S. Li, H. Kitamura, Y. (2019) Cryogenic-based CO2 capture technologies: State-of-the-art developments and current challenges, Renewable and. Sustainable Energy Review. 101. 265-278. http://doi.org/10.1016/j.rser.2018.11.018.

[22] Khalilpour, R. Mumford, K. Zhai,H. Abbas, A. Stevens, G. Rubin, E. (2015) Membrane-based carbon capture from flue gas: A review, Journal of Cleaner Production 103. 286-300. http://doi.org/10.1016/j.jclepro.2014.10.050.

[23] Favre, E. Bounaceur, R. Roizard, D. (2009) Biogas, membranes and carbon dioxide capture, Journal of Membrane Science 328 (1) 11-14. http://doi.org/10.1016/j.memsci.2008.12.017.

[24] Chuah, C. Goh, K. Yang, Y. Gong, H. Li,W. H. Karahan, E. et al., (2018) Harnessing filler materials for enhancing biogas separation membranes, Chem. Rev. 118 (18) 8655-8769. http://doi.org/10.1021/acs.chemrev.8b00091.

[25] Hidalgo, D. Sanz-Bedate, S. Martín-Marroquín, J. Castro, J. Antolín, G.(2020) Selective separation of $\mathrm{CH} 4$ and $\mathrm{CO} 2$ using membrane contactors, Renew. Energy. 150 935-942. http://doi.org/10.1016/j.renene.2019.12.073.

[26] Powell, C. Qiao, G (2006) Polymeric CO2/N2 gas separation membranes for the capture of carbon dioxide from power plant flue gases, Journal of Membrane Science 279 (1-2) 1-49. http://doi.org/10.1016/j.memsci.2005.12.062.

[27] Jamil, A. Ching, O. Shariff, P. (2014) Polymer-nanoclay mixed matrix membranes for $\mathrm{CO} 2 / \mathrm{CH} 4$ separation: A review, Applied Mechanics and Materials. 625. 690-695. http://doi.org/10.4028/www.scientific.net/AMM.625.690.

[28]Khaleque, A. Alam, M.M. Hoque, M. Mondal, S. Bin Haider, Xu, Bet al., (2020) Zeolite synthesis from low-cost materials and environmental applications: A review, Environmental Advances. 2. 100019. http://doi.org/10.1016/j.envadv.2020.100019.

[29] Richter, H. Reger-Wagner, N. Kämnitz, S Voigt, I. Lubenau, U. Mothes, (2019) R. Carbon membranes for bio gas upgrading, Energy Procedia. 158 (2018) 861-866. http://doi.org/10.1016/j.egypro.2019.01.222.

[30] Koros, W.J. Mahajan, R. (2001) Pushing the limits on possibilities for large scale gas separation: Which strategies?. Journal of Membrane Science 181 (1) 141. http://doi.org/10.1016/S03767388(00)00676-1.

[31] Burggraaf, A.J.(1999) Single gas permeation of thin zeolite (MFI) membranes: theory and analysis of experimental observations, Journal of Membrane Science 155 (1) 45-65. http://doi.org/10.1016/S0376-7388(98)00295-6.

[32] Kujawa, J. Cerneaux, S Kujawski, W. (2015) Removal of hazardous volatile organic compounds from water by vacuum pervaporation with hydrophobic ceramic membranes, Journal of Membrane Science 474 (2015) 11-19. http://doi.org/10.1016/j.memsci.2014.08.054.

[33] Wang, K. Lin, X. Jiang, G. Liu, L. Jiang, L. Doherty, C. et al., (2014). Slow hydrophobic hydration induced polymer ultrafiltration membranes with high water flux, Journal of Membrane Science 471 (2014) 27-34. http://doi.org/10.1016/j.memsci.2014.07.073.

[34] Lay, W. Zhang, J. Tang, C. Wang, R. Liu, Y. Fane, A. (2012). Factors affecting flux performance of forward osmosis systems, Journal of Membrane Science 394-395. 151-168. http://doi.org/10.1016/j.memsci.2011.12.035.

[35] Caro, J. Noack, M. (2008). Zeolite membranes \pm state of their development and perspective, Microporous and Mesoporous Materials. 115 (3) 215-233. http://doi.org/ 10.1016/j.micromeso.2008.03.008

[36] Vanherck, K. Koeckelberghs, G. Vankelecom, I. (2013). Crosslinking polyimides for membrane applications: A review, Progress in Polymer Sciences 38 (6) 874-896. http://doi.org/ 10.1016/j.progpolymsci.2012.11.001.

[37] Deng, L. Xue, Y. Yan, J. Lau, C. Cao, B. Li, P. (2019). Oxidative crosslinking of copolyimides at sub-Tg temperatures to enhance resistance against $\mathrm{CO} 2$-induced plasticization, Journal of Membrane Science 583. 40-48. http://doi.org/10.1016/j.memsci.2019.04.002.

[38] Shah, M. McCarthy, M. Sachdeva, S. Lee, A. Jeong, H. (2012). Current status of metal-organic framework membranes for gas separations: Promises and challenges, Industrial and Engineering Chemistry Research. 51 (5) 2179-2199. http://doi.org/10.1021/ie202038m.

[39] Rangnekar,N. Mittal, N. Elyassi, B. Caro, J. Tsapatsis, M. (2015) Zeolite membranes - a review and comparison with MOFs, Chem. Soc. Rev. 44 (20) 7128-7154. http://doi.org/10.1039/c5cs00292c.

[40] Chung, S. Park, J. Li, D. Ida, J. Kumakiri, I. Lin, J. (2005). Dual-phase metal-carbonate 
membrane for high-temperature carbon dioxide separation, Industrial and Engineering Chemistry Research. 44 (21) 7999-8006. http://doi.org/10.1021/ie0503141.

[41] Wang, B. Sheng, M. Xu, J. Zhao, S.Wang, J. Wang, Z. (2020) Recent Advances of Gas Transport Channels Constructed with Different Dimensional Nanomaterials in Mixed-Matrix Membranes for CO2 Separation, Small Methods. 4 (3) 1-16. http://doi.org/10.1002/smtd.201900749.

[42] Sun, H. Wang,T. Xu, Y.Gao, W. Li, P. Niu, Q. (2017) Fabrication of polyimide and functionalized multi-walled carbon nanotubes mixed matrix membranes by in-situ polymerization for $\mathrm{CO} 2$ separation, Separation and Purification Technology $177 . \quad 327-336$. http://doi.org/10.1016/j.seppur.2017.01.015.

[43] Qiao, Z. Zhao, S.Wang, J. Wang, S.Wang, Z. Guiver, M. (2016) A Highly Permeable Aligned Montmorillonite Mixed-Matrix Membrane for CO2 Separation, Angewandte Chemie International Edition 55 (32) 9321-9325. http://doi.org/10.1002/anie.201603211.

[44] Wang, B. Xie, L.Wang, X. Liu, X. Li, J. J (2018). Applications of metal-organic frameworks for green energy and environment: New advances in adsorptive gas separation, storage and removal, Green Energy Environmental. 3 (3) 191-228. http://doi.org/10.1016/j.gee.2018.03.001.

[45] Scholes, C. Stevens, G. Kentish, S. (2012). Membrane gas separation applications in natural gas processing, Fuel. 96 (2012) 15-28. http://doi.org/10.1016/j.fuel.2011.12.074.

[46] Caro, J. (2020). Diffusion coeficcients in nanoporous solids derived from membrane permeation measurements. The Journal of Membrane Biology. 583. 1-2. https://doi.org/10.1007/s10450-02000262-z.

[47] Wijmans, J. Baker, R. (1995) The solution-diffusion model: a review, Journal of Membrane Science 107 (1-2) 1-21. https://doi.org/10.1016/0376-7388(95)00102-I.

[48] Benito, J. Conesa, A. Rodriguez, M. ((2004). Membranas cerámicas: Tipos, metodos de obtención t caracterización. Cerámica y Vidrio, 43 (5) 829-842.

[49] Ruthven, D. DeSisto, W. Higgins, S. (2009). Diffusion in a mesoporous silica membrane: Validity of the Knudsen diffusion model, Chemical Engineering Science 64 (13) 3201-3203. https://doi.org/10.1016/j.ces.2009.03.049.

[50] Hernandez, A. Calvo, J. Prfidanos, P. Tejerina, F. (1996). Pore size distributions in microporous membranes. A critical analysis of the bubble point extended method, Journal of Membrane Science. 7388. 1-12. https://doi.org/10.1016/0376-7388(95)00025-9

[51] Izquierdo, M. (2008). Temperature influence on transport parameters characteristic of Knudsen and Poiseuille flows, Chemical Engineering Science. 63 (22) 5531-5539. https://doi.org/10.1016/j.ces.2008.07.034.

[52] Scholes, C. (2018). Water resistant composite membranes for carbon dioxide separation from methane, Applied Science 8 (5). 2-12. https://doi.org/10.3390/app8050829.

[53] Lei, L. Lindbråthen, A. Zhang, X Favvas, E. Sandru, Hillestad M., et al., (2020). Preparation of carbon molecular sieve membranes with remarkable $\mathrm{CO} 2 / \mathrm{CH} 4$ selectivity for high-pressure natural gas sweetening, Journal of Membrane Science 614 (7491) 118529. https://doi.org/10.1016/j.memsci.2020.118529.

[54] Jomekian, A. Bazooyar,B. Behbahani,R. (2020). Experimental and modeling study of CO2 separation by modified Pebax 1657 TFC membranes, Korean Journal Chemical Engineering . 37 (11) 2020-2040. https://doi.org/10.1007/s11814-020-0598-y.

[55] Chen, X. Rodriguez, D. Kaliaguine, S. (2012). Diamino-organosilicone APTMDS: A new crosslinking agent for polyimides membranes. Separation and Purification Technology. 86 (2012) 221-233. doi:10.1016/j.seppur.2011.11.008.

[56] Vaughn, J. Koros, W. (2012). Effect of the amide bond diamine structure on the CO2, H2S, and $\mathrm{CH} 4$ transport properties of a series of novel 6FDA-based polyamide-imides for natural gas purification, Macromolecules. 45 (17) 7036-7049. https://doi.org/10.1021/ma301249x.

[57] Ahmad, A. Adewole, J. Leo, C. Sultan, A. S. Ismail, S. (2014) Preparation and gas transport properties of dual-layer polysulfone membranes for high pressure $\mathrm{CO} 2$ removal from natural gas, J. Appl. Polym. Sci. 131 (20) 1-10. https://doi.org/10.1002/app.40924.

[58] Hossain, I. Nam, C.Rizzuto, S. Barbieri, G. Tocci, E. Kim, T. (2019). PIM-polyimide multiblock copolymer-based membranes with enhanced $\mathrm{CO} 2$ separation performances, Journal of Membrane Science 574. 270-281. https://doi.org/:10.1016/j.memsci.2018.12.084.

[59] Li, X. Wang, Y Wu, Y. Song, S. Wang, B. Zhong, S. et al., High-performance SSZ-13 membranes prepared using ball-milled nanosized seeds for carbon dioxide and nitrogen separations from methane, Chinese Journal Chemical Engineering. 28 (5) 1285-1292. https://doi.org/10.1016/j.cjche.2020.02.004.

[60] Wang, B. Gao, F. Zhang, F. Xing, W. Zhou, W. (2019). Highly permeable and oriented AIPO- 
18 membranes prepared using directly synthesized nanosheets for $\mathrm{CO} 2 / \mathrm{CH} 4$ separation, Journal of Material Chemistry A. 7 (21) 13164-13172. https://doi.org/10.1039/c9ta01233h.

[61] Liu, B. Zhou, R. Yogo, K Kita, H. (2019). Preparation of CHA zeolite (chabazite) crystals and membranes without organic structural directing agents for $\mathrm{CO} 2$ separation, Journal of Membrane Science 573. 333-343. https://doi.org/10.1016/j.memsci.2018.11.059.

[62] Yu, L.Fouladvand, S. Grahn, M. Hedlund, J. (2019) Ultra-thin MFI membranes with different $\mathrm{Si} / \mathrm{Al}$ ratios for CO2/CH4 separation, Microporous Mesoporous Materials. 284 (2019) 258-264. https://doi.org/10.1016/j.micromeso.2019.04.042.

[63] Hayakawa, E. Himeno S. , Synthesis of all-silica (2020). ZSM-58 zeolite membranes for separation of $\mathrm{CO} 2 / \mathrm{CH} 4$ and $\mathrm{CO} 2 / \mathrm{N} 2$ gas mixtures, Microporous and Mesoporous Materials. 291. 109695. https://doi.org/:10.1016/j.micromeso.2019.109695.

[64] Sen, M. Dana, K. Das, N. (2018). Development of LTA zeolite membrane from clay by sonication assisted method at room temperature for $\mathrm{H} 2-\mathrm{CO} 2$ and $\mathrm{CO} 2-\mathrm{CH} 4$ separation, Ultrason. Sonochem. 48 (2018) 299-310. https://doi.org/10.1016/j.ultsonch.2018.06.007.

[65] Rad, M. Fatemi, S. Mirfendereski, S. (2012). Development of T type zeolite for separation of CO2 from $\mathrm{CH} 4$ in adsorption processes, Chemical Eng. Res. Des. 90. 1687-1695. https://doi.org/10.1016/j.cherd.2012.01.010.

[66] Mofarahi, M. Gholipour, F.(2014) Gas adsorption separation of $\mathrm{CO} / \mathrm{CH} 4$ system using zeolite $5 \mathrm{~A}$, Microporous and Mesoporous Materials. 200 (2014) 1-10. https://doi.org/10.1016/j.micromeso.2014.08.022.

[67] Ahmad, M. Martin-Gil,V. Supinkova, T. Lambert, R. Castro-Muñoz, R. Hrabanek, P. et al., (2021) Novel MMM using CO2 selective SSZ-16 and high-performance 6FDA-polyimide for $\mathrm{CO} 2 / \mathrm{CH} 4$ separation, Separation and Purification Technology. $254 . \quad 117582$. https://doi.org/10.1016/j.seppur.2020.117582.

[68] Park, S. Bang, J. Choi, J. Lee, S. Lee, J. Lee, J. (2014) 3-Dimensionally disordered mesoporous silica (DMS)-containing mixed matrix membranes for $\mathrm{CO} 2$ and non-CO2 greenhouse gas separations, Separation and Purification Technology 136. 286-295. https://doi.org/10.1016/j.seppur.2014.09.016.

[69] Zhao, J Xie,K. Liu, L Liu, M. Qiu, W. Webley, P. (2019). Enhancing plasticization-resistance of mixed-matrix membranes with exceptionally high $\mathrm{CO} 2 / \mathrm{CH} 4$ selectivity through incorporating ZSM-25 zeolite, Journal of Membrane https://doi.org/10.1016/j.memsci.2019.03.073.

[70] Li, W. Chuah, C. Nie, L. Bae, T. (2019). Enhanced CO2/CH4 selectivity and mechanical strength of mixed-matrix membrane incorporated with NiDOBDC/GO composite, Journal of Industrial and Engineering Chemistry. 74. 118-125. https://doi.org/10.1016/j.jiec.2019.02.016.

[71] Farashi, Z. Azizi, S. Rezaei-Dasht Arzhandi, M. Noroozi, Z. Azizi, N. (2019). Improving $\mathrm{CO} 2 / \mathrm{CH} 4$ separation efficiency of Pebax-1657 membrane by adding Al2O3 nanoparticles in its matrix, Journal of Natural Gas Science and Engineering. 72.103019. https://doi.org/10.1016/j.jngse.2019.103019.

[72] Shin, H. Chi, W. Bae, S. Kim, J. J. Kim,(2017). High-performance thin PVC-POEM/ZIF-8 mixe Journal of Industrial and Engineering Chemistry. 53 (2017) 127-133. https://doi.org/10.1016/j.jiec.2017.04.013.

[73] Guo, A. Ban, Y. Yang, K. Yang, W.(2018). Metal-organic framework-based mixed matrix membranes: Synergetic effect of adsorption and diffusion for $\mathrm{CO} / \mathrm{CH} 4$ separation, Journal of Membrane Science 562. 76-84. doi:10.1016/j.memsci.2018.05.032.

[74] Ebadi Amooghin, A. Omidkhah, M. Kargari, A. (2015). The effects of aminosilane grafting on $\mathrm{NaY}$ zeolite-Matrimid®5218 mixed matrix membranes for $\mathrm{CO} 2 / \mathrm{CH} 4$ separation, Journal of Membrane Scence. 490. 364-379. https://doi.org/10.1016/j.memsci.2015.04.070.

[75] Gong, H. Lee, S. Bae, T. (2017). Mixed-matrix membranes containing inorganically surfacemodified 5A zeolite for enhanced $\mathrm{CO} 2 / \mathrm{CH} 4$ separation, Microporous an Mesoporous Materials 237. 82-89. https://doi.org/10.1016/j.micromeso.2016.09.017. 